\title{
Control algorithm for a residential photovoltaic system with storage
}

\author{
Yannick Riesen $^{\mathrm{a}, 1, *}$, Christophe Ballif ${ }^{\mathrm{a}}$, Nicolas Wyrsch ${ }^{\mathrm{a}}$ \\ ${ }^{a}$ Ecole Polytechnique Fédérale de Lausanne (EPFL), Institute of Microengineering (IMT), Photovoltaics and Thin-Film Electronics Laboratory, Rue de la \\ Maladière 71, CH-2000 Neuchâtel, Switzerland
}

\begin{abstract}
High penetration of photovoltaic (PV) electricity could affect the stability of the low-voltage grid due to over-voltage and transformer overloading at times of peak production. Residential battery storage can smooth out those peaks and hence contribute to grid stability. A feed-in limit allows for the easy setting of a maximum power injection cap and motivates PV owners to increase their self-consumption. A simple control strategy for a residential battery system coupled with a PV system that maximizes selfconsumption and minimizes curtailment losses due to a feed-in limit is presented. The algorithm used in this strategy does not require a forecast of insulation conditions. The performance of this algorithm is compared to a second algorithm-a control strategy based on linear optimization using a forecast. Assuming an exact forecast, this second algorithm is very close to the maximum self-consumption and minimum curtailment losses achievable and can be used to benchmark the simple strategy. The results show that the simple strategy performs as well as the second algorithm with exact forecasts and performs significantly better than the second algorithm using real forecasts. Moreover, it is shown that this result is valid for a large range of storage capacities and PV sizes. Furthermore, it is shown that with a time resolution of 15 minutes for the input data (the resolution of most PV production and load data) self-consumption is overestimated by about $3 \%$ and curtailment losses are underestimated by the same amount. Load sensitivity simulations show that different load curve shapes do not fundamentally change the results. Finally, to assess the effect of load aggregation, the case where the strategy is applied separately to 44 households with storage is compared to the case where it is applied to a centralized storage system of the same size as the total storage of the 44 households. The reduction of the curtailment losses with the number of aggregated houses is showed.
\end{abstract}

Keywords: Photovoltaic (PV), Home storage system, Battery management strategy, PV integration into the electrical grid

\section{Highlights}

- A control strategy for a battery system coupled with a PV system is presented.

- With a feed-in limit this strategy does not need a PV production forecast.

- This strategy performs as well as a strategy relying on an exact forecast.

- A relatively small storage size allows peak injection reduction of $50 \%$.

\section{Introduction}

Residential electric energy storage systems coupled with a photovoltaic (PV) installation could contribute to the stability of the low-voltage grid in the case of high PV penetration by absorbing the production power peaks around midday

\footnotetext{
${ }^{*}$ Corresponding author

Email addresses: yannick.riesen@alumni.epfl.ch (Yannick Riesen), christophe.ballif@epfl.ch (Christophe Ballif), nicolas.wyrsch@epfl.ch (Nicolas Wyrsch)

${ }^{1}$ Present address: Planair SA, Crêt 108a, CH-2314 La Sagne, Switzerland

$[1,2,3,4,5]$. Moreover, such a system increases PV selfconsumption, which can provide an economic benefit to the system owner due to lower electricity exchange with the grid and minimized electricity transport losses $[6,7,8]$. Economic assessment of such systems optimizing only self-consumption can be found in [9, 10, 11]. In Germany, financial incentives for battery storage are available provided that the feed-in power is limited to $50 \%$ of the PV system's nominal power [12]. By 2015, more than 12'000 such systems were installed in Germany [13]. As shown by [14], active power curtailment allows for stabilizing the grid voltage. Imposing a feed-in limit is a simple and efficient method to avoid high injection peaks and to hence minimize grid disturbances allowing for higher PV penetration $[15,16]$. However, this limit induces curtailment losses even in the presence of energy storage systems. Therefore, control strategies that minimize those losses and maximize self-consumption are needed. Alternative strategies than a fixed feed-in limit prevent injection peaks are described in $[17,18,14]$.

Several control strategies that allow for the efficient shaving of injection peaks and the maximization of self-consumption at the same time have been proposed in the literature. Solutions based on exact (or perfect) forecasts are presented in [19, 20]. However, forecast inaccuracies induce non-negligible changes in the performance of the systems [21, 22, 23]. To circumvent 
those issues, strategies that do not need forecasts were developed. Zeh et al. [22] proposed a feed-in damping strategy, in which the battery is charged using a nearly constant power defined by the battery capacity divided by the time until sunset. This approach gives better results than a feed-in chopping strategy which starts to charge only when a daily maximum feed-in power calculated with a forecast is reached. Moshövel et al. [23] used a persistence forecast strategy based on the optimal state of charge of the previous day. This approach resulted in a self-consumption value $4.4 \%$ lower compared to the one with a perfect forecast.

In this paper, in contrast to our previous work [24] and [19, 20], a new control strategy that does not need forecast data and is valid only in the presence of a feed-in limit is developed. The novelty of the solution presented here compared to, e.g. [22, 23] is that clear sky production data is used, which can be easily simulated. This data allows for a more precise control strategy allowing for self-consumption close to its maximum value and a reduction of the curtailment losses.

The system under consideration and the corresponding simulation program that was developed is described. In addition to the control algorithm that does not need forecast data, a control strategy that maximizes financial cash flow due to electricity exchange with the grid is introduced and discussed. This second algorithm requires production and consumption forecasts. It is used to benchmark the first algorithm. Both strategies are evaluated in the frame of a feed-in limit and for the second algorithm using a real or an exact (perfect) forecast as a function of the battery capacity, the PV sizing and the value of the feed-in limit.

Most load profiles and PV production data are available with a resolution of 15 minutes. The time resolution of the input data affects the simulation results such as self-consumption share and the assessment of the effects on the grid [25, 26]. Therefore, the effect of the input data resolution ( 5 seconds to 30 minutes) on the self-consumption and loss due to the feed-in limit using the algorithm that does not need forecast data is evaluated. The effect of this time resolution and of its optimal choice is discussed.

To assess the sensitivity of the results as a function of the load curve shape, the same algorithm is applied to 44 different real loads of households recorded in a small Swiss town. Finally, using those 44 loads, we quantify the gain in peak shaving and self-consumption by aggregating loads.

\section{Methods}

\subsection{System configuration}

There are two main system configurations for a PV system coupled with a battery: the DC-link configuration, in which the battery is connected before the DC/AC converter, and the AClink configuration, in which the battery is connected through a bidirectional AC/DC inverter directly to the AC home grid $[27,6]$. The choice of configuration does not significantly change self-consumption simulation results, and the DC-link configuration is used here. Like in the study by Magnor et al.
[28], an energy-flow model is applied in this work. Note also that, within this study, power flow from the grid to the battery is not allowed.

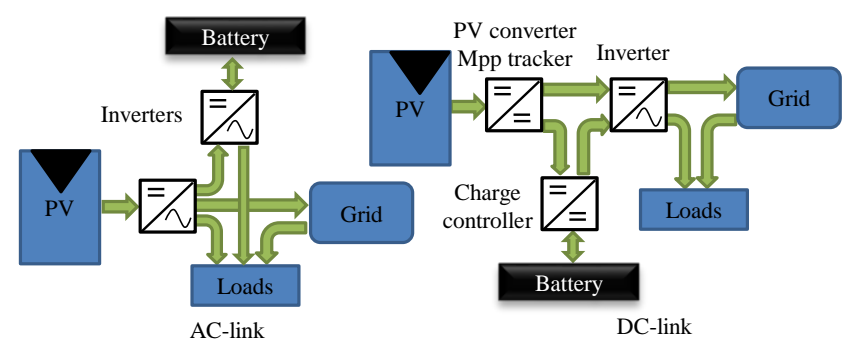

Figure 1: Layouts of PV-systems coupled with a battery storage in the AC-and DC-link configuration.

The efficiency values as a function of input power of the DCDC converter and the DC-AC inverter are calculated according to typical curves of commercially available systems [29]. If not stated differently, the converter and inverter nominal power is equal to the DC nominal power of the PV installation. The temperature and voltage dependence of the inverter efficiency are neglected; its efficiency depends only on the input power. A simple battery model with a fixed round-trip efficiency of 90 $\%$ is used, which is representative of standard Li-ion batteries. The choice of a Li-ion battery is motivated by the potentially lower cycle cost in the long term due to a higher cycle number and lifetime [30].

The battery storage capacity is defined as the effective capacity. For example, a battery with $10 \mathrm{kWh}$ storage capacity and a minimal recommended state of charge of $20 \%$ has an effective capacity of $8 \mathrm{kWh}$.

\subsection{Description of the control algorithms}

In this work, two control algorithms that optimize financial balance (cost minimization) with regard to electricity exchange with the grid (buying or selling electricity, cash flow) are studied. In the presence of a feed-in limit, this objective is equivalent to first minimizing the losses due to feed-in power curtailment and then enhancing self-consumption (as long as the feed-in tariff $\geq 0$ and $<$ electricity price). The two control algorithms are:

- Cost minimization without a forecast (co. opt. w/o forecast,), requiring only clear sky PV production data. This algorithm implements the simple strategy that we propose, which is applicable only if a feed-in limit exists.

- Cost minimization based on PV production and the load forecast using a linear optimization (co. opt. re. forecast with real forecast). Using an exact forecast (co. opt. ex. forecast), this algorithm gives results close to the best financial balance (discrepancies arise due to the lower time resolution used for the optimization - 30 minutes - compared to the simulation time resolution -1 or 15 minutes) 


\author{
Nomenclature \\ $B^{+}$Battery charging energy at time t \\ $B^{-}$Battery discharging energy at time $\mathrm{t}$ \\ $B_{\text {loss }}$ Battery losses \\ $B_{\text {cap }}$ Battery capacity \\ $F^{+}$Feed-in tariff \\ $F^{-}$Retail electricity price \\ FiL feed-in limit \\ $g f$ Power exchanged with the grid \\ $L_{t}$ Load at time $t$ \\ $L_{\text {tot }}$ Total load energy \\ $M E P$ Maximum excess PV energy \\ $P V_{t}$ PV production at time $\mathrm{t}$ \\ $P V_{c s}$ Simulated clear sky PV production
}

$P V_{t o t}$ Total PV production

$S O C$ State of charge of the storage

$t$ Time step

max. sc. Maximization of self-consumption

min. curt. loss. Minimization of curtailment losses

co. opt. w/o forecast Cost minimization without forecast

co. opt. re. forecast Cost minimization with exact forecast using linear optimization

co. opt. re. forecast Cost minimization with real forecast us-

ing linear optimization

curt. loss. Curtailment losses

SC Self-consumption

C Cashflow

PV Photovoltaic and can be used to benchmark the co. opt. w/o forecast algorithm.

Those strategies are useful only if the battery cannot absorb the complete daily PV production. To further benchmark the effectiveness of the algorithms, two more control algorithms are introduced:

- Maximization of self-consumption (max. sc.). The battery is charged (if the state of charge allows) as soon as the PV power exceeds the load (or consumption) and is discharged as soon as the PV power is lower than consumption. This strategy is often implemented in simple home storage systems.

- Minimization of PV production losses due to the feed-in limit (min. curt. loss.). Excess PV power that would be curtailed by the feed-in limit is stored (if the state of charge allows) in the battery. The battery is discharged as soon as PV power is lower than consumption. This strategy is applicable only in the presence of a feed-in limit.

\subsubsection{Cost minimization without a forecast}

This simple algorithm (co. opt. w/o forecast) optimizes the financial balance due to grid exchange in the presence of feedin limits and constant electricity/feed-in prices without the need for a forecast. This algorithm needs only the theoretical clear sky maximum PV production curve to minimize curtailment loss and to maximize self-consumption. For this purpose, the maximum clear sky PV production is calculated for each minute using the PV lib toolbox [31]. The maximum excess PV energy $M E P$ is the energy necessary to absorb the clear sky PV production power that is higher than the feed-in limit:

$$
M E P_{t}=\left.\int_{t+1}^{10 \text { hours }}\left(P V_{c s, t}-F i L\right) \cdot d t\right|_{P V_{c s}-F i L>0}
$$

with $P V_{c s}$ the simulated clear sky PV production, FiL the feedin limit and $t$ the time step. The excess PV energy is calculated for the next 10 hours as, for each considered day in Switzerland, the period of excess PV production is shorter than 10 hours. For places with longer daylight, this time span should be increased. The battery is controlled as follows and is summarized with an if/then flowchart in Figure 2:

- Excess PV power that would be curtailed by the feed-in limit is stored (if the state of charge allows) in the battery. Moreover, if the battery's available capacity is sufficient to absorb the maximum excess PV production of the next 10 hours (battery storage capacity $B_{\text {cap }}$ minus the state of charge $S O C$ is higher than the maximum excess energy, $B_{\text {cap }}-S O C>M E P$ ), the battery is charged until this limit.

- If PV power is higher than the load power (gridflux $g f=$ $P V-L<0)$, the battery is charged only if its capacity is sufficient to absorb the maximum excess PV energy $(M E P)$.

- If PV power is lower than the load power $(g f<0)$, the battery is discharged.

- The system boundaries must be satisfied (battery capacity, inverter power limits).

\subsubsection{Cost minimization with forecast}

This control algorithm optimizes the gain or cost (co. opt. forecast) due to exchange with the grid (selling or buying electricity) and depends on feed-in tariffs and electricity costs. This algorithm is valid with and without the feed-in limit and needs forecasts. A schematic representation can be found in Figure 3. This algorithm was introduced in a previous study by Riesen $e t$ al. [24]. 


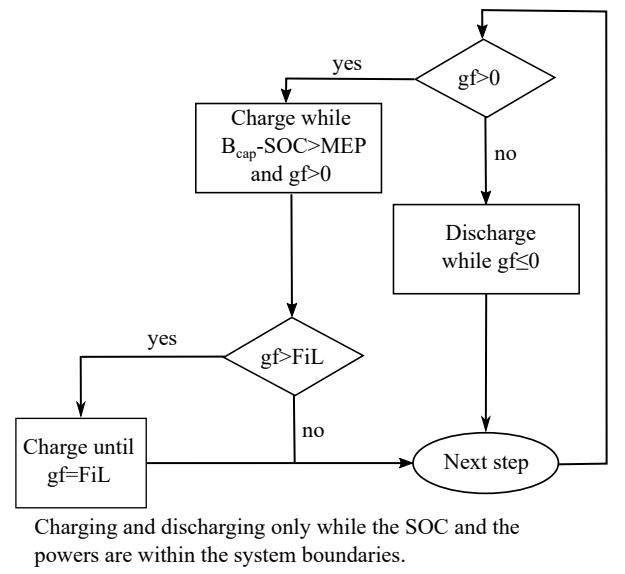

Figure 2: If/then flowchart of the co. opt. w/o forecast strategy, with SOC the state of charge, $B_{c a p}$ the storage capacity, $F i L$ the feed-in limit, $g f=P V_{t}-$ $L_{t}$ the power exchange with the grid (gridflux) which is the PV production $P V_{t}$ minus the load $L_{t}$ at the actual time step $t$ and $M E P$ the maximum excess energy.

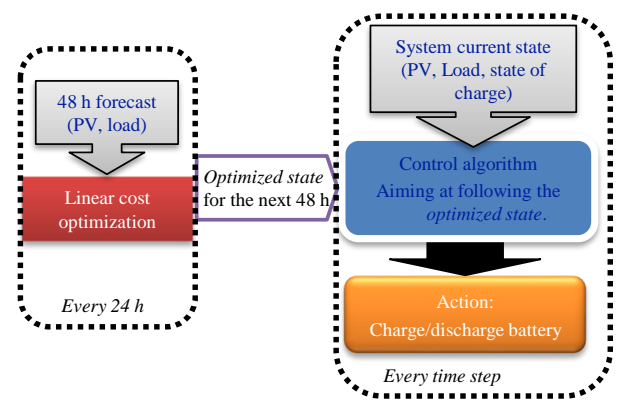

Figure 3: Schematic representation of the cost minimization algorithm with forecast (co. opt. forecast) algorithm.

Every 24 hours, a cost optimization of the energy flux to or from the battery is done according to the forecast data for the next 48 hours of PV production (see subsection 2.2.5), household load and buying/selling prices. The energy flux is then converted to an optimal state of charge for each simulation time step (in our case 1 or 15 minutes). For this optimization, a linear programming algorithm is applied because of faster computing and a guarantee to find the global optimum. A description can be found in the appendix 7 . The inverter and converter efficiencies are considered as constant (not dependent on the input power) to allow a linear optimization. This is considered to be an acceptable approximation as the power intensities that contribute the most to the energy fluxes are at high input powers where the efficiency of the inverter is relatively constant (with input power). Moreover, this algorithm also allows us to add an additional cost that is proportional to the daily maximum injected power to the objective function, a power tariff. The effect of this tariff is the minimization of the injected power value into the grid. This functionality is not used in this work but is mentioned in the appendix.

At each simulation time step, the control algorithm defines the battery flux in order to reach the optimized state of charge if allowed by the following rules:

- The resulting energy fluxes have to respect the different limits of the system (inverter power limit, power flux limits of the battery, storage capacity).

- If there is a feed-in limit, the PV power exceeding that limit is stored as long as the previously cited constraints are met disregarding the optimal state of charge (SOC). If this is not possible, this excess PV power (defined as curt. loss.) is lost. In practice, this means that the maximum power tracker of the PV system is no longer operated at maximum power point .

\subsubsection{Maximization of self-consumption}

This algorithm (max. sc.) charges the battery (if the state of charge allows) as soon as the PV power exceeds the load (or consumption) and discharges it as soon as the PV power is lower than consumption.

\subsubsection{Minimization of $P V$ curtailment losses}

With this algorithm (min. curt. loss.), the excess electricity resulting from PV power being curtailed by the feed-in limit is stored in the battery (if the state of charge allows). The battery is discharged as soon as the PV power is lower than consumption.

\subsubsection{Simulation data}

The following load profiles are used:

Reference load is based on data measured at one-minute time steps of a Swiss household of five people (one family house with central oil heating), from April, 2012, to March, 2013, located near Neuchâtel. The annual load consumption was $4943 \mathrm{kWh}$.

High time resolution load is based on data measured at fivesecond time steps of the same household as the reference load, from September, 2014, to September, 2015.

15 min loads are based on data measured with smart meters at 15-minute time steps in a town in the canton of Neuchâtel from April, 2012, to March, 2013. The data comprise representative loads of family houses and apartments. Figure 4 (left) shows the sum of all 44 loads for a day in March. We see the typical residential load shape with relatively low consumption at night and two peaks at midday and in the evening. On the right, a histogram of the annual electricity consumption shows that most of the households consume around $4 \mathrm{MWh} /$ year.

The PV production curve was generated with the PV-lib toolbox [31] assuming a multi-crystalline silicon module taken from the PV-lib toolbox library and a module tilt angle of $30^{\circ}$. Note, that for this study, the method of PV production does not play a crucial role. As weather input data measured global horizontal irradiance and temperature values from two different sources were used: 


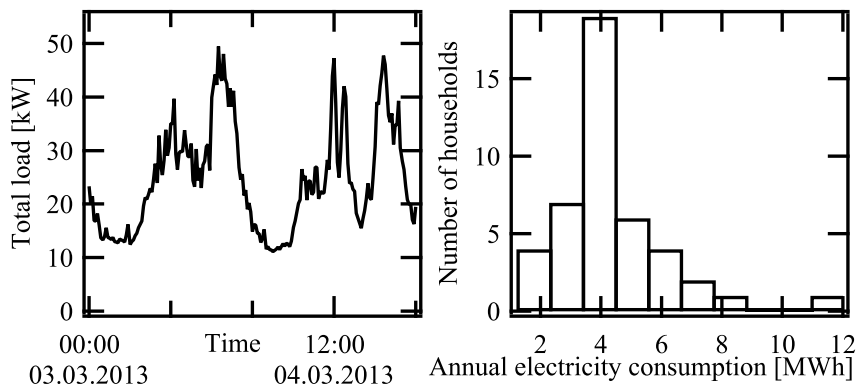

Figure 4: (Left) Sum of the 4415 min loads for 48 hours in March, 2013. (Right) Histogram of the annual electricity consumption of the 15 min loads.

- The MeteoSwiss station in Neuchâtel with data recorded at 10-minute time steps at the same time as the available forecast data (April, 2012, to March, 2013). For the same time period, the real PV forecast is based on historical forecast data from Meteotest (48 hours ahead in one-hour time steps). The forecasts were interpolated to 30-minute time step data. Note that a lower time step would slow down the optimization, and the forecast accuracy would not really benefit from it.

- A weather station located on the roof of our institute with data recorded at five-second time steps. These data, recorded from September, 2014, to September, 2015, were used for the time resolution sensitivity simulations (see section 3.2).

The resulting module power was then interpolated or integrated to the needed simulation time step.

The load forecast was generated by averaging for each 30 minutes the historical load profiles of each day of the week for each season. During days when a household was unoccupied, a special holiday average load curve was generated.

To compare the effect of forecast errors, an exact forecast having the same format as the real forecast, but using the effective production and loads, is introduced. Hence, in this case, there are no forecast errors (exact forecast).

\section{Results}

\subsection{Comparison of the different control algorithms with a feed- in limit}

The different control strategies are here applied to the reference load and the MeteoSwiss weather data to evaluate their performance in reducing PV production loss due to the feed-in limit (curt. loss.), maximizing self-consumption and optimizing cash flow.

\subsubsection{Effects of the storage size}

Figure 5 shows curt. loss. and self-consumption for a feedin-limit of $50 \%$ as a function of the battery capacity using the reference load.

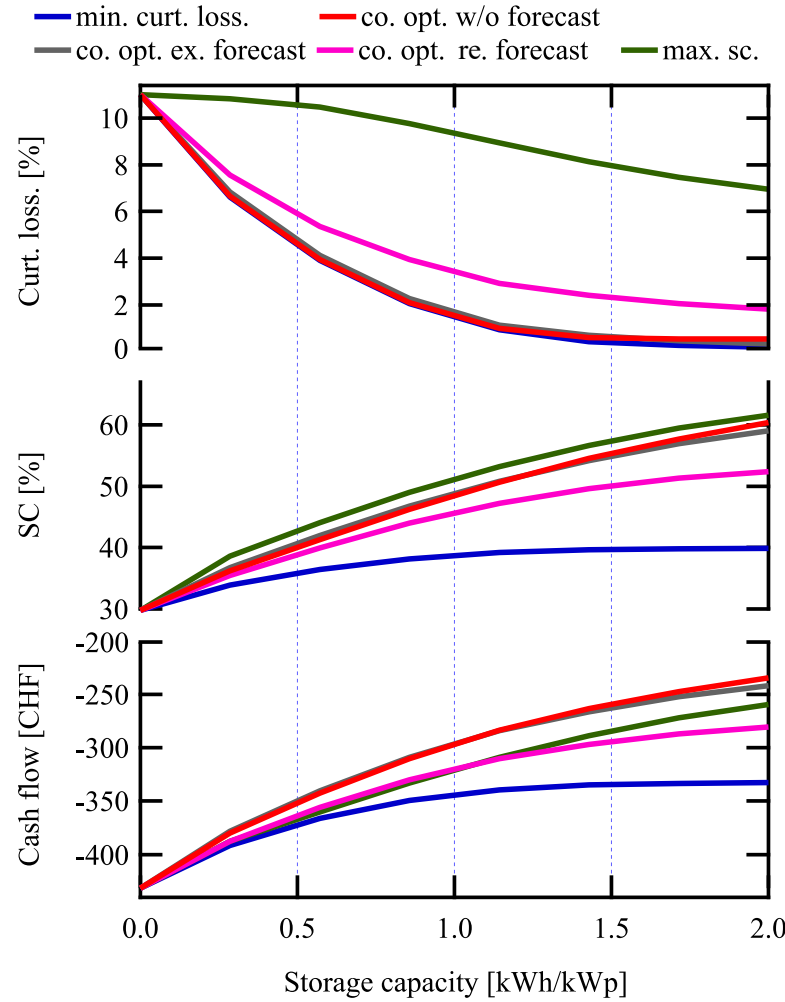

Figure 5: (Top) PV production loss due to the feed-in limit (curt. loss.), (middle) self-consumption (SC) and (bottom) cash flow for a single household (reference load) using the different battery control strategies as a function of storage capacity with a feed-in limit of $50 \%$.

The curt. loss. values are, unsurprisingly, the smallest for the min. curt. loss. algorithm. Up to a battery capacity of 1 $\mathrm{kWh} / \mathrm{kWp}$, the co. opt. w/o forecast algorithm has a curt. loss. value almost as low as that of the optimum. For higher storage capacities, the curtailment loss of the co. opt. w/o forecast deviates slightly from the optimum. This is due to the assumption that the battery is always empty before the first grid injection, which is not always the case for high battery capacity. Hence the available storage capacity is overestimated, resulting in higher curtailment loss. For storage capacities above 2 $\mathrm{kWh} / \mathrm{kWp}$, the difference in the curt. loss. values between the co. opt. w/o forecast and the min. curt. loss. algorithms remains constant. As the discharging power of the battery is limited by the DC/AC inverter, increasing its nominal power could, to a limited extent, empty the battery faster during peak consumption and slightly diminish the curtailment loss for high storage capacities. Theoretically, the co. opt. ex. forecast should give the same curt. loss. values as the min. curt. loss. case. This is almost the case: the differences are due to the different time steps of the optimization algorithm (30 minutes) and the simulation time step ( 1 minute) and the constant inverter efficiency approximation which results in slightly higher curtailment loss for the co. opt. ex. forecast case. With real forecasts (co. opt. re. forecast), the curt. loss. values are higher; for example, with a storage capacity of $1 \mathrm{kWh} / \mathrm{kWp}$, the curt. loss. is more 
than two times higher than for min. curt. loss.. Those higher losses arise on days when the forecasted amount of excess PV is underestimated and therefore the storage is already full when it is needed to absorb excess power.

Self-consumption increases from $30 \%$ without a battery to over $60 \%$ for storage capacities above $1.7 \mathrm{kWh} / \mathrm{kWp}$. The selfconsumption of the co. opt. ex. forecast approach is almost as high as that of the best case ( $\max$. sc.). However, the co. opt. re. forecast strategy with real forecasts has lower self-consumption than the two others due to days when the PV production is overestimated. The difference can reach $7 \%$ absolute. In terms of self-consumption, the co. opt. w/o forecast algorithm performs as well as the co. opt. ex. forecast algorithm even for storage capacities much higher than those shown in Figure 5. If the battery can store the total PV production that cannot be locally consumed, the co. opt. w/o forecast strategy behaves in a similar way as the max. sc. strategy.

As any charging to or discharging from the grid is forbidden, the curtailment loss and self-consumption can be linked to the financial balance due to grid exchange (cash flow). Given the $S C$ (eq. 2) and the $C u r t_{\text {loss }}$ (eq. 3) the cash flow $C$ for any feedin tariff $F^{+}$and the retail electricity price $F^{-}$can be calculated:

$$
\begin{aligned}
S C & =\frac{\sum_{t} L_{t}+\left.\sum_{t}\left(P V_{t}-L_{t}+B^{+}\right)\right|_{\left(P V_{t}-L_{t}\right)<0}}{\sum_{t} P V_{t}} \\
C u r t_{\mathrm{loss}} & =\frac{\left.\sum_{t}\left(P V_{t}-L_{t}+B^{-}\right)\right|_{\left(P V_{t}-L_{t}+B^{-}\right)>F_{\mathrm{limit}}}}{\sum_{t} P V_{t}} \\
C & =\left.\sum_{t}\left(P V_{t}-L_{t}+B^{-}\right)\right|_{\left(P V_{t}-L_{t}\right)>0} \cdot F^{+} \\
& +\left.\sum_{t}\left(P V_{t}-L_{t}+B^{+}\right)\right|_{\left(P V_{t}-L_{t}\right)<0} \cdot F^{-} \\
& =S C \cdot P V_{\mathrm{tot}}\left(F^{-}-F^{+}\right)+P V_{\mathrm{tot}}\left(1-\text { Curt }_{\mathrm{loss}}\right) \cdot F^{+} \\
& -B_{\mathrm{loss}} F^{+}-L_{\mathrm{tot}} \cdot F^{-}
\end{aligned}
$$

where $L_{t}$ is the load at time $\mathrm{t}, P V_{t}$ is the $\mathrm{PV}$ production at time $\mathrm{t}, B^{+}$is the battery charging energy at time $\mathrm{t}$ (positive value), $B^{-}$is the battery discharging energy at time t (negative value), $B_{\text {loss }}=\sum B^{+}+\sum B^{-}$is the battery losses and is equal to the difference between the total battery charging and discharging energy, $L_{t o t}=\sum L_{t}$ and $P V_{t o t}=\sum P V_{t}, S C$ the self-consumption and $C u r t_{\text {loss }}$ the curt. loss.. The variation in $C(\Delta C)$ can be written as a function of $S C$ and the curt. loss. variation (due to e.g. a new battery capacity) can be written as eq. 8 :

$$
\Delta C=P V_{\text {tot }}\left(\Delta S C \cdot\left(F^{-}-F^{+}\right)-\Delta C u r t_{\text {loss }} \cdot F^{+}\right)-\Delta B_{\text {loss }} F^{+}
$$

In the case studied here, with $\left(F^{+}<F^{-}\right)$, in eq. 8 it is seen that the $S C$ value mainly influences the cash flow. By setting the retail electricity price to $F^{-}=20 \mathrm{cts} / \mathrm{kWh}$ and the feed-in tariff to $F^{+}=10 \mathrm{cts} / \mathrm{kWh}$, the results shown in Figure 5 (bottom) are obtained. The co. opt. ex. forecast and co. opt. $w / o$ forecast algorithms gave almost the same results and performs best. At storage capacities higher than $1.5 \mathrm{kWh} / \mathrm{kWp}$ the co. opt. w/o forecast strategy performs even slightly better, because the bigger the storage the better this algorithm will perform. In addition because of the time resolution difference between the optimization (30 minutes) and the simulation step
(1 minute), the co. opt. ex. forecast strategy does not reach the self-consumption optimum. With a storage capacity of 2 $\mathrm{kWh} / \mathrm{kWp}$ the annual electricity cost can be reduced by $45 \%$ from $431 \mathrm{CHF}$ to $197 \mathrm{CHF}$. Due to the higher curt. loss. and lower self-consumption, the co. opt. re. forecast algorithm is financially less favorable than the co. opt. ex. forecast and co. opt. $w / o$ forecast algorithms. The financial gain on the electricity cost is reduced to $34 \%$. As no exact forecast is possible, the simple co. opt. w/o forecast control algorithm is the best choice and will be used for the next simulations presented in this work. This result is true independently of pricing as long as the electricity retail price is higher than the feed-in tariff and the prices are constant in time.

With a further increase of the storage capacity, the curt. loss. for the max. $s c$. algorithm will continue to decrease, increasing the cash flow gain. At the same time the self-consumption of the co. opt. w/o forecast strategy will reach the optimum as there will be more than enough battery capacity to charge the excess energy, hence the battery will be charged as soon as there is injection. This will result, for high battery capacity, that the cash flow of the max. sc. will reach the one of the co. opt. w/o forecast strategy but not will not be better.

\subsubsection{Effects of the feed-in limit and PV-sizing}

Using the same inputs as in sec. 3.1.1, the storage capacity is fixed to $1 \mathrm{kWh} / \mathrm{kWp}$ and varied:

- the PV system size with an annual PV production covering $0.33 \%$ to $200 \%$ of the consumption and a fixed feed-in limit of $50 \%$.

- the feed-in limit from $0 \%$ (no injection into the grid) to $100 \%$ with an annual PV production covering the consumption.

Figure 6 (left) shows self-consumption as a function of PV sizing and (right) curt. loss. as a function of the feed-in limit.

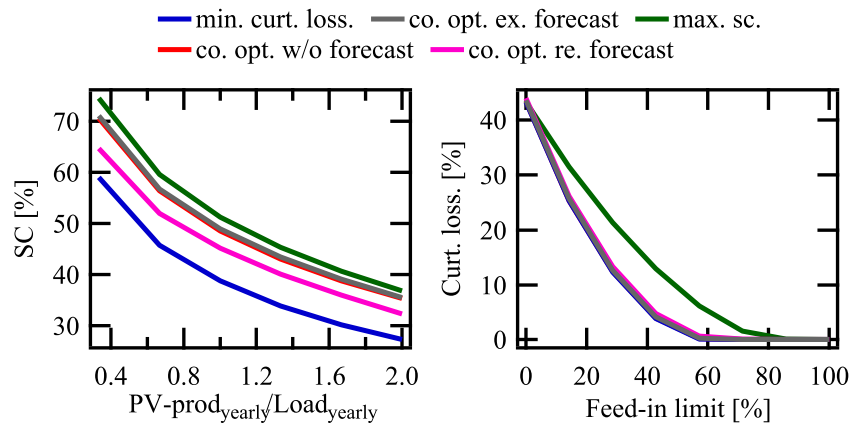

Figure 6: (left) Self-consumption (SC) as a function of the PV sizing and (right) curtailment loss as a function of the feed-in limit for the different control strategies.

The self-consumption value decreases from $60 \%$ to $73 \%$ (depending on the control strategy) for a PV sizing that covers $33 \%$ of the yearly load to values between $28 \%$ and $38 \%$ for a PV sizing that covers two times the annual consumption. 
For the whole PV sizing range, the different self-consumption values as a function of the control strategy behave similarly. Hence the same conclusions as in section 3.1.1 can be applied, namely that the co. opt. w/o forecast algorithm yields a selfconsumption value as high as the co. opt. ex. forecast algorithm. The same is true for the curtailment loss curves (not shown).

With a storage capacity of $1 \mathrm{kWh} / \mathrm{kWp}$, more than $40 \%$ of the PV production is lost if injection is prohibited (feed-in limit $=0 \%$ ) (Figure 6, right). With the exception of the max. sc. control strategy, all strategies attain the same curt. loss. values.

\subsection{Effect of the time resolution}

Most load profile data available have a time step of $15 \mathrm{~min}$ utes. However, the duration of peak consumption is often shorter. Depending on meteorological conditions, e.g. for partly cloudy conditions, production may vary significantly within a period of a few seconds. The effect of time resolution on the curtailment loss is evaluated using a feed-in limit of $50 \%$ and no battery. For this purpose, the high time resolution load and the weather data measured on the roof of our institute were used. Figure 7 shows curtailment loss as a function of the time resolution in the cases where only production, only electrical load and both time resolutions are varied from 5 seconds to 30 minutes. The time resolution of the parameter that is not varied is set to the lowest value of 5 seconds.
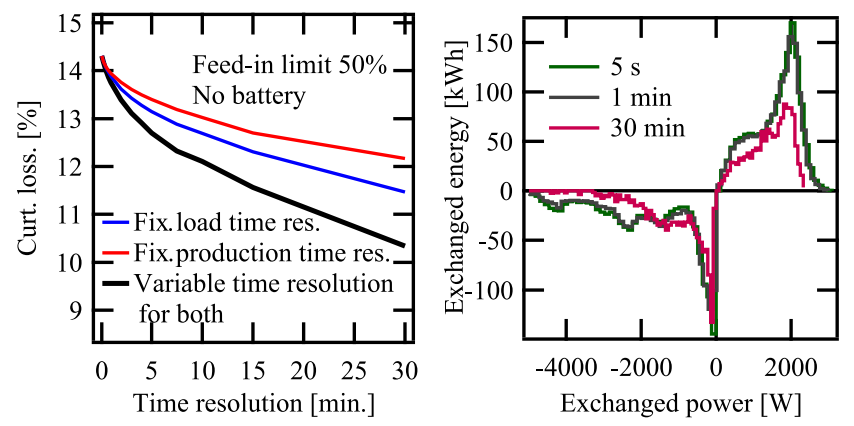

Figure 7: (Left) Curtailment loss as a function of the time steps when only production, only consumption or both are varied with time steps from 5 seconds to 30 minutes. (Right) Histogram of the annually cumulated energy for power steps of $75 \mathrm{~W}$ exchanged with the grid.

Figure 7 (left) shows that by increasing the time step from 5 seconds to 1,15 and 30 minutes, the curtailment losses are underestimated by respectively $0.6 \%, 2.7 \%$ and $4.0 \%$ in absolute values. The PV production time resolution contributes slightly more than the consumption time resolution to this underestimation. The histogram-like Figure 7 (right) shows the yearly exchanged energy with the grid as a function of the exchanged power intensity. The highest and lowest exchanged power values are lower for the curve with highest time steps (30 minutes), which reflects the smoothing out of the power peaks induced by the reduction of the resolution. Moreover, the higher the time step, the lower the energy exchanged with the grid, due to the summing of the positive and negative power exchange with the grid within a time step.

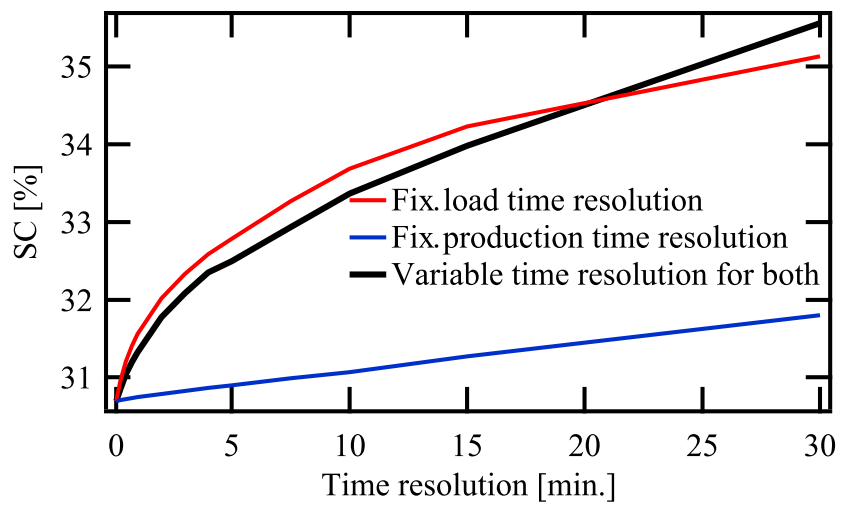

Figure 8: Self-consumption (SC) as a function of the time resolution of the loads, PV production and both.

Figure 8 shows self-consumption as a function of time resolution. By increasing the time resolution to 15 minutes, selfconsumption is overestimated by $3 \%$. The fixed production time resolution curve induces the lowest errors. The two other curves induce higher errors which are similar for both. With a relative increase of the self-consumption of $16 \%$ by increasing the granularity from 10 seconds to six minutes, values similar to those of Beck et al. [25] (between $5 \%$ and $20 \%$ ) are obtained. However, in their case, the slope of the self-consumption curve increases with higher time steps, while in our case it decreases. At this point no explanation is found for this discrepancy. However, the results of this work are in agreement with our previous paper [32] using different input data.

The time resolution influences only the simulation results but has no effect on the control strategies. Therefore, comparison of performances of the algorithms should be assessed using similar time resolutions for the input data. As shown in [32], adding storage reduces the errors introduced by longer time resolutions. In this case, the results shown in Figure 8 represents the upper limit of these errors.

\subsection{Load sensitivity}

Up to now the algorithms were applied to only one household using the reference load. In this section, the co. opt. w/o forecast simple control algorithm is applied to 44 different loads (15 min loads) for a load sensitivity study. The feed-in limit is fixed to $50 \%$ and the PV installation nominal power is fixed such that the yearly PV-produced energy is equal to the total annual consumption for each of the loads.

Figure 9 shows the maximum, minimum and mean values of the curtailment loss (left) and self-consumption (right) as a function of the storage capacity. Overall, the same behaviors as for the reference load are observed. All households are within $\mathrm{a} \pm 2.5 \%$ band for curtailment loss and $\mathrm{a} \pm 6 \%$ band for the self-consumption.

\subsection{Local and district storage}

In this section the gain of applying the control algorithm to aggregated loads is compared to the case where all households 

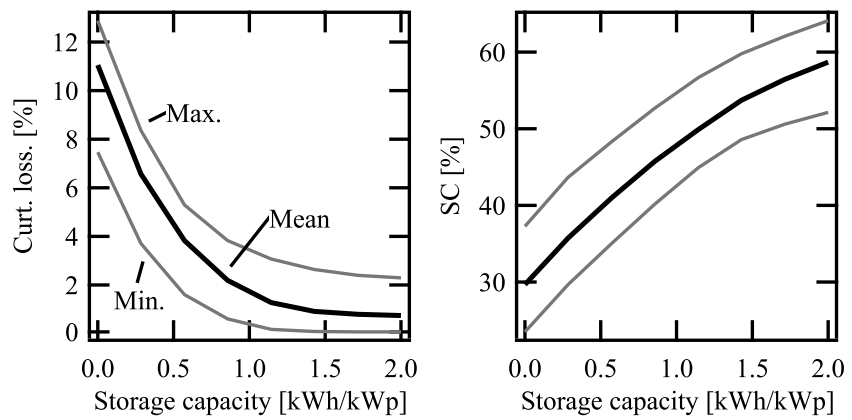

Figure 9: Maximum, minimum and mean of the curtailment loss and selfconsumption (SC) as a function of the storage capacity divided by the nominal PV power for 44 different loads (apartments and family houses) with a feed-in limit of $50 \%$.

have their own individual batteries and apply the optimization only for themselves (distributed optimization). Aggregated loads means that the feed-in limit is applied to the sum of the loads and that either all batteries are centrally controlled or that there is one central battery. The co. opt. w/o forecast control algorithm is applied to the $4415 \mathrm{~min}$ loads and the feed-in limit is again set to $50 \%$. The yearly PV-produced energy equals the total annual consumption.

In Figure 10 left the curt. loss. is plotted as a function of the number of aggregated loads (15 min loads, chosen randomly). For both relative storage capacities, the curt. loss. decays rapidly by more than $0.4 \%$ absolute up to four aggregated households and then continues to decay slightly with fluctuations. A decaying trend is expected in all cases. However, depending on the randomly chosen loads, the curt. loss. can locally fluctuate with increasing aggregation. The small peaks seen in Figure 10 left illustrate this fact. The self-consumption as a function of loads remains more or less stable and varies according to the mean of the individual self-consumption.
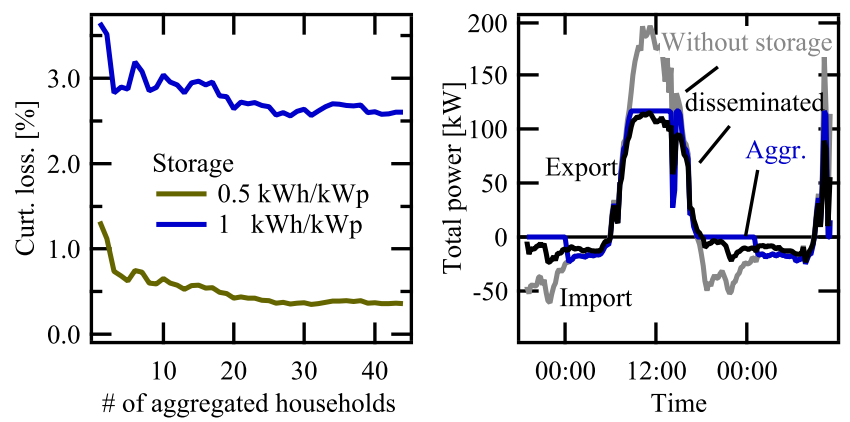

Figure 10: (Left) Curtailment loss as a function of the number of aggregated loads for two different storage capacities based on a one-year simulation (Right) Electricity flux from/to the grid for one sunny day for the case of no storage (grey), disseminated storage (black) and central storage (blue).

For comparison, the co. opt. w/o forecast control algorithm is also applied individually to the 44 different loads and PV sizes and to the case of a PV field that has the same total energy production as the cumulative PV production of all loads and a centralized storage (all 44 loads aggregated). The total PV production is equal to the total yearly consumption (190'000 $\mathrm{kWh}$ ). For this comparison a storage size of $1 \mathrm{kWh} / \mathrm{kWp}$ and a feed-in limit of $50 \%$ are used. Figure 10 (right) shows the flux with the grid for an almost clear sky day. The yearly curt. loss., self-consumption and cash flow based on are presented in Table 1. The curt. loss. decreases while self-consumption

\begin{tabular}{lcc}
\hline & Disseminated & centralized \\
\hline curt. loss. & $1.6 \%$ & $0.4 \%$ \\
SC & $49.8 \%$ & $51.3 \%$ \\
Cash flow & $-11525 \mathrm{CHF}$ & $-10750 \mathrm{CHF}$ \\
\hline
\end{tabular}

Table 1: Comparison of curtailment loss, self-consumption and cash flow between disseminated and centralized PV and storage.

increases with centralized PV and storage (aggregated). The higher the load intensity compared to the nominal PV power, the lower will be the curt. loss. decrease by aggregating the loads. Note that, on a local level, only the load - and not the PV production - is smoothed by aggregation as the same irradiation for all modules is assumed.

In the presence of a feed-in limit, aggregation of loads allows for reducing the curt. loss. and in some cases enhancing the self-consumption. This is demonstrated with a feed-in limit of $50 \%$; however, the same behavior is expected for other values. Moreover, in addition to the more favorable cash flow, running, maintenance and investment costs should be lower for centralized storage compared to distributed storage.

\section{Discussion}

The main results of this study are further discussed in this section and summarized in Table 2.

\subsection{Power flow model}

A power flow model and a simple battery model was used to keep the results as general as possible. The validity of this approach was discussed in [28]. The conclusion of the control strategy comparison should not be significantly different when using a more accurate and detailed model. This is also true for the evaluation of curtailment loss. Self-consumption may be affected by the model accuracy as it depends on the different components (battery type, inverter type) of the system and hence the calculated value can vary from case to case. However, system performance analysis for a given installation should take into account voltages and currents as well as a more precise battery model to get more accurate results.

\subsection{Performance of the cost minimization without forecast}

The developed control strategy requiring no forecast (co. opt. $w / o$ forecast) is able to minimize curt. loss. reaching close to optimum values. This is the case because enough battery capacity is always kept free such that the excess energy (with higher power than the feed-in limit, $M E P$ ) can be stored in the case of highest PV production. With self-consumption, the situation 


\begin{tabular}{|c|c|}
\hline Subject & Main results \\
\hline Best algorithm (sec. 3.1) & $\begin{array}{l}\text { The co. opt. w/o forecast strategy give a self-consumption value } \\
\text { as high and as low curt. loss. as the co. opt. forecast ex. algo- } \\
\text { rithm. }\end{array}$ \\
\hline $\begin{array}{l}\text { Effect of the time resolu- } \\
\text { tion (sec. } 3.2 \text { ) }\end{array}$ & $\begin{array}{l}\text { By varying the time resolution from } 5 \text { seconds to } 15 \text { minutes using } \\
\text { the co. opt. w/o forecast algorithm, the curt. loss. decreases by } \\
2.7 \% \text { and self-consumption increases by } 3 \%\end{array}$ \\
\hline Load sensitivity (sec. 3.3) & $\begin{array}{l}\text { By Applying the co. opt. w/o forecast strategy to } 44 \text { different load } \\
\text { curves with a feed-in limit of } 50 \% \text {, curt. loss. (self-consumption) } \\
\text { varies in a band of } \pm 2.5 \%( \pm 6 \%) \text {. }\end{array}$ \\
\hline $\begin{array}{l}\text { Local vs. district storage } \\
\text { (sec. 3.4) }\end{array}$ & $\begin{array}{l}\text { Aggregation reduces essentially the } \text { curt. loss. between } 0.4 \% \text { and } \\
1 \% \text { absolute }\end{array}$ \\
\hline
\end{tabular}

Table 2: Summary of the results with a feed-in limit of $50 \%$

is different. The worst cases happen on days with low irradiation (e.g. overcast), in which the battery should be charged as soon as there is power injection into the grid. However, with the co. opt. w/o forecast strategy, battery charging can be delayed (because of the need to keep clear sky production capacity reserve $M E P$ ). As a consequence, there may be days in which the battery is not filled to the maximum possible state at sunset. However, along the day, as the $M E P$ diminishes and the state of charge remains low, the battery will charge more as sunset approaches. Hence the discrepancy to the optimal case still remains relatively low (around $2 \%$ absolute of self-consumption). This discrepancy is close to the value of $4.4 \%$ obtained with the persistence forecast strategy used in [23]. Note that the bigger the battery size (compared to the feed-in limit), the nearer the performance will reach the optimum. Therefore, these results are valid for a broad range of storage capacity, PV sizes and feed-in limit values.

As shown in Table 2, the co. opt. w/o forecast strategy gives a self-consumption value as high, and a low curt. loss. value as low as the co. opt. forecast ex. algorithm, which is near to the optimal and much better than the algorithm with real forecasts. Hence, even if the co. opt. re. forecast and co. opt. ex. forecast control strategies are improved (for example by optimizing every hour and not every 24 hours), with a real forecast it will barely reach the performances with an exact forecast and of the simple algorithm without a forecast. Therefore in the presence of a fixed feed-in limit, a forecast is not needed to achieve high self-consumption and low curt. loss. .

\subsection{Variable feed-in limit}

The co. opt. w/o forecast strategy can also be used with a variable feed-in limit. The feed-in limit could, for example, be defined for each day as the maximum feed-in limit such that all PV excess energy could be absorbed by the system. In this case, however, a forecast is needed to determine this maximum.

\subsection{Effect of time resolution}

It was shown that, with a resolution of 15 minutes for input data, the curtailment loss is underestimated by more than $2 \%$ absolute and self-consumption is overestimated by more than 3
$\%$ absolute, compared to a simulation with five-second resolution. Those results are based on one load profile and could be slightly different depending on the load profile shape and the weather conditions.

\subsection{Load aggregation}

When the control algorithm is applied to the sum of several loads, only one central battery or distributed batteries with a central control can be used. Aggregating loads mainly diminishes the curtailment loss; the value of this diminution depends on the ratio between the load and the PV production. Moreover, in real conditions, one centralized battery should be more costeffective than distributed systems. However, one possible disadvantage of a centralized battery could be the loss of concern of the households. Decentralized PV and storage incites people to self-consume as much as possible. This incentive disappears with centralization if no other incentive scheme is proposed.

\section{Conclusion}

Adding storage significantly reduces curtailment losses due to a feed-in limit. A control strategy for a battery coupled with a PV system that, in the presence of a feed-in limit, minimizes the losses due this limit and maximizes self-consumption was developed. This simple strategy requires the maximum PV production only under clear sky conditions. The three main advantages of this strategy are its simplicity requiring very limited computing power, the absence of communication required with the outside of the household, and its independence from forecast errors. It performs almost as well as a strategy with a linear optimization and exact forecasts that is near to the theoretical best case and much better than this algorithm with real forecasts. For example, with a feed-in limit of $50 \%$ of the nominal PV power (as is present in Germany), with no batteries about 7 $\%$ to $12 \%$ of the total PV power has to be curtailed and is lost, as shown for a sample of 44 representative loads of households. Adding storage and applying the developed control strategy significantly reduces those losses. For example, a battery capacity of $1 \mathrm{kWh} / \mathrm{kWp}$ reduces those losses to values of $0.5 \%$ to $4 \%$. Hence a relatively small storage capacity already allows us to divide the maximum injected power value by two (by setting a 
feed-in limit of $50 \%$ ) with very low losses and allows for high PV penetration into the electrical grid. Those results demonstrate that, in the presence of a given feed-in limit no forecast is needed to minimize curt. loss. and, maximize self-consumption and financial gains due to energy flow to/from the grid.

By applying the control strategy without forecast to quantify the effect of time resolution of the input data on the results, it was shown that higher time steps induce errors of a few percent in absolut on self-consumption and curt. loss.. Moreover by applying, this algorithm to aggregated loads, the advantage of using load aggregation in the framework of feedin limits which mainly allows to reduce the curtailment losses was demonstrated. Finally, a feed-in limit could also be used to avoid or reduce power fees as applied in some locations. Our results could also be applied in such cases.

\section{Acknowledgements}

This work was supported by EOS Holding; by the Swiss Federal Office of Energy in the framework of the Flexi Project; and by the CTI in the framework of the SCCER Furies project.

\section{Appendix}

\subsection{Linear optimization}

The PV system coupled with a battery presented in Section 2 is simplified such that we can find an optimum battery control using linear programming. The objective is financial optimization with regard to electricity exchange with the grid. We use the Matlab linear programming solver which finds the minimum of the following problem:

$$
\min _{x} f^{T} x \text { such that }\left\{\begin{array}{l}
A \cdot x \leq b \\
A e q \cdot x=b e q \\
l b \leq x<u b
\end{array}\right.
$$

In order to implement the different constraints such as the different power limits and the battery size we defined the different parameters of eq. 9 accordingly. $n$ is the number of time steps.
We have:

$$
x=\left(\begin{array}{c|c}
G_{1}^{+} & \text {(flux to the grid) } \\
\vdots & \vdots \\
G_{n}^{+} & \\
G_{1}^{-} & \text {(flux from the grid) } \\
\vdots & \vdots \\
G_{n}^{-} & \\
B_{1}^{+} & \text {(flux to the battery) } \\
\vdots & \vdots \\
B_{n}^{+} & \\
B_{1}^{-} & \text {(flux from the battery) } \\
\vdots & \vdots \\
B_{n}^{-} & \\
I_{1}^{+} & \text {(flux to the inverter DC side) } \\
\vdots & \vdots \\
I_{n}^{+} & \\
I_{1}^{-} & \text {(flux to the inverter AC side) } \\
\vdots & \vdots \\
I_{n}^{-} & \\
R_{1} & \text { (proportion of the PV power actually used) } \\
\vdots & \vdots \\
R_{n}
\end{array}\right) \text { }
$$

The vector $x$ also contains an extra variable, which is required to be larger than the flow to the grid at any time. It can be used to compute a power-dependent price in the objective function.

We define $\vec{P}$ as the PV production, $\vec{L}$ the load, $E B$ the battery efficiency and $E I$ the inverter efficiency with the assumption that it is constant. There are two global fluxes:

$$
\begin{array}{r}
0=-R \cdot P+B^{+}-E B \cdot B^{-}+I^{+}-E I \cdot I^{-} \\
L=-G^{+}+G^{-}-I^{-}+E I \cdot I^{+}
\end{array}
$$

We implement these constraints using the second linear programming constraint $A e q \cdot x=b e q$. Therefore we have:

Aeq $=\left(\begin{array}{ccccccc}0 \cdot I_{n} & 0 \cdot I_{n} & I_{n} & -E B \cdot I_{n} & 1 \cdot I_{n} & -E I \cdot I_{n} & -P \cdot I_{n} \\ -1 \cdot I_{n} & 0 \cdot I_{n} & 0 \cdot I_{n} & 0 \cdot I_{n} & E I \cdot I_{n} & -1 \cdot I_{n} & 0 \cdot I_{n} \\ 0 \cdot \overrightarrow{1} & 0 \cdot \overrightarrow{1} & \overrightarrow{1} & 0 \cdot \overrightarrow{1} & 0 \cdot \overrightarrow{1} & 0 \cdot \overrightarrow{1} & 0 \cdot \overrightarrow{1}\end{array}\right)$

With the $n \times n$ identity matrix:

$$
I_{n}=\left(\begin{array}{cccc}
1 & 0 & \ldots & 0 \\
0 & 1 & \ldots & 0 \\
\vdots & \vdots & \ddots & \vdots \\
0 & 0 & \ldots & 1
\end{array}\right)
$$


and $\overrightarrow{1}=\left(\begin{array}{llll}1 & 1 & \ldots & 1\end{array}\right)$ with size of $n$. For beq we have:

$$
\text { beq }=\left(\begin{array}{c}
0 \cdot \overrightarrow{1}^{\prime} \\
L_{1} \\
\vdots \\
L_{n}
\end{array}\right)
$$

The constraints $A x<b$ from 1 to $n$ implement the constraints implied by the maximal capacity of the battery. The similar constraints from $n+1$ to $2 n$ implement the constraints implied by the fact that the battery cannot be negatively charged. We have:
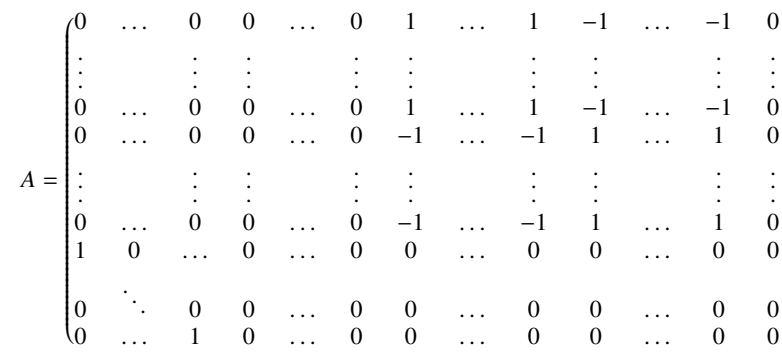

For $b$ :

$$
b=\left(\begin{array}{c}
B C \\
\vdots \\
B C-\text { Bini } \\
\text { Bini } \\
\vdots \\
\text { Bini } \\
0 \\
\vdots \\
0 \\
\vdots
\end{array}\right)
$$

With $B C$ the battery capacity and Bini the initial battery capacity.

The boundaries of the power fed to the battery and going through the inverter are set through $l b$ and $u b$ vectors (see eq. 9).
Finally for the objective function we define:

$$
f=\left(\begin{array}{c}
-s_{1} \\
\vdots \\
-s_{n} \\
b_{1} \\
\vdots \\
b_{n} \\
0 \\
\vdots \\
\vdots \\
0 \\
d \\
\vdots \\
d \\
P T
\end{array}\right)
$$

With $s_{i}$ and $b_{i}$ electricity selling and buying price, $P T$ the power tariff and $d$ a constant with a relatively low value to favor reducing the PV power instead of dissipating energy by having both incoming and outgoing fluxes in the battery and the inverter. A positive value of $P T$ corresponds to a tax that is relative to the highest injected power. Hence the battery is charged with some peak shaving even in the absence of a feed-in limit.

\section{References}

[1] K. Büdenbender, M. Braun, A. Schmiegel, D. Magnor, J.-C. Marcel, Improving pv-integration into the distribution grid contribution of multifunctional pv-battery systems to stabilised system operation, in: 25th European Photovoltaic Solar Energy Conference and Exhibition, 2010

URL http://www.sol-ion-project.eu/export/sites/ default/en/_data/publications/mediatheque-files/Conf_ EUPVSEC_Valencia_IWES_0910.pdf

[2] A. Schmiegel, K. Koch, A. Meissner, P. Knaup, C. Jehoulet, H. Schuh, M. Landau, M. Braun, K. Büdenbender, R. Geipel, C. Vachette, D. Sauer, D. Magnor, J.-C. Marcel, The sol-ion system, an integrated pv-system with lithium ion batteries system performance, in: 25th European Photovoltaic Solar Energy Conference and Exhibition, 2010.

URL http://www.sol-ion-project.eu/export/sites/ default/en/_data/publications/mediatheque-files/Conf_ EUPVSEC_Valencia_Voltwerk_0910.pdf

[3] M. Lodl, R. Witzmann, M. Metzger, Operation strategies of energy storages with forecast methods in low-voltage grids with a high degree of decentralized generation, in: 2011 IEEE Electrical Power and Energy Conference, Institute of Electrical \& Electronics Engineers (IEEE), 2011. doi:10.1109/epec.2011.6070252.

URL http://dx.doi.org/10.1109/EPEC. 2011.6070252

[4] J. von Appen, A. Schmiegel, M. Braun, Impact of pv storage systems on low voltage grids: A study on the influence of pv storage systems on the voltage symmetry of the grid, in: 27th European Photovoltaic Solar Energy Conference, 2012.

[5] C. Bucher, G. Andersson, L. Küng, Increasing the pv hosting capacity of distribution power grids: a comparison of seven methods, in: EUPVSEC Paris, 2013.

[6] R. Luthander, J. Widén, D. Nilsson, J. Palm, Photovoltaic selfconsumption in buildings: A review, Applied Energy 142 (2015) 80-94. doi: 10.1016/j. apenergy .2014.12.028.

URL http://dx.doi.org/10.1016/j.apenergy .2014.12.028 
[7] Y. Riffonneau, S. Bacha, F. Barruel, S. Ploix, Optimal power flow management for grid connected pv systems with batteries, Sustainable Energy, IEEE Transactions on 2 (3) (2011) 309-320. doi : 10.1109/TSTE. 2011.2114901.

[8] M. Braun, K. Büdenbender, D. Magnor, A. Jossen, Photovoltaic selfconsumption in germany - using lithium-ion storage to increase selfconsumed photovoltaic energy, in: 24th EuropeanPhotovoltaic SolarEnergy Conference, 21-25 September 2009, Hamburg, Germany, 2009.

[9] C. S. Lai, M. D. McCulloch, Levelized cost of electricity for solar photovoltaic and electrical energy storage, Applied Energy 190 (2017) 191 - 203. doi:http://dx.doi.org/10.1016/j.apenergy . 2016. 12.153.

URL http://www.sciencedirect.com/science/article/pii/ S030626191631933X

[10] B. Numbi, S. Malinga, Optimal energy cost and economic analysis of a residential grid-interactive solar $\{\mathrm{PV}\}$ system- case of ethekwini municipality in south africa, Applied Energy 186, Part 1 (2017) 28 - 45. doi:http://dx.doi.org/10.1016/j.apenergy.2016.10.048.

URL http://www.sciencedirect.com/science/article/pii/ S0306261916314921

[11] S. Quoilin, K. Kavvadias, A. Mercier, I. Pappone, A. Zucker, Quantifying self-consumption linked to solar home battery systems: Statistical analysis and economic assessment, Applied Energy 182 (2016) 58 - 67. doi:http://dx.doi.org/10.1016/j.apenergy.2016.08.077. URL http://www.sciencedirect.com/science/article/pii/ S0306261916311643

[12] K.-P. Kairies, D. Haberschusz, J. van Ouwerkerk, J. Strebel, O. Wessels, D. Magnor, J. Badeda, D. U. Sauer, Wissenschaftliches messund evaluierungsprogramm solarstromspeicher, Tech. rep., Institut f?r Stromrichtertechnik und Elektrische Antriebe der RWTH Aachen (2016). URL http://www.speichermonitoring.de/fileadmin/user upload/Speichermonitoring_Jahresbericht_2016_Kairies_ web.pdf

[13] 35 prozent mehr solarstromspeicher, Online, accessed 28.08.2015, http://die-sonne-speichern.de/pressemeldungen/35-prozent-mehrsolarstromspeicher/.

[14] J. von Appen, T. Stetz, M. Braun, A. Schmiegel, Local voltage control strategies for pv storage systems in distribution grids, IEEE Transactions on Smart Grid 5 (2) (2014) 1002-1009. doi : 10.1109/TSG . 2013. 2291116.

[15] J. Weniger, J. Bergner, D. Beier, M. Jakobi, T. Tjarko, V. Quaschning, Grid feed-in behavior of distributed pv battery systems, in: 31st European Photovoltaic Solar Energy Conference and Exhibition. WIP-Renewable Energies, Munich, Germany, 2015, pp. 1726-1732.

[16] C. Gaudin, A. Ballanti, E. Lejay, Evaluation of pv curtailment option to optimize pv integration in distribution network, in: CIRED Workshop Lisbon 29-30 May 2012, IET, IET, 2012.

[17] M. Kim, S. Bae, Decentralized control of a scalable photovoltaic (pv)-battery hybrid power system, Applied Energy 188 (2017) 444 - 455. doi:http://dx.doi.org/10.1016/j.apenergy.2016.12.037. URL http://www.sciencedirect.com/science/article/pii/ S0306261916318001

[18] M. Resch, J. Bühler, M. Klausen, A. Sumper, Impact of operation strategies of large scale battery systems on distribution grid planning in germany, Renewable and Sustainable Energy Reviews 74 (2017) 1042 1063. doi:http://dx.doi.org/10.1016/j.rser.2017.02.075 URL http://www.sciencedirect.com/science/article/pii/ S1364032117302976

[19] J. Li, M. A. Danzer, Optimal charge control strategies for stationary photovoltaic battery systems, Journal of Power Sources 258 (2014) 365-373. doi:10.1016/j.jpowsour.2014.02.066.

URL http://dx.doi.org/10.1016/j.jpowsour.2014.02.066

[20] M. Schneider, P. Boras, H. Schaede, L. Quurck, S. Rinderknecht, Effects of operational strategies on performance and costs of electric energy storage systems, Energy Procedia 46 (2014) 271-280. doi:10.1016/j . egypro.2014.01.182.

URL http://dx.doi.org/10.1016/j.egypro.2014.01.182

[21] E. Lorenz, J. Remund, S. Müller, W. Traunmüller, G. Steinmaurer, D. Pozo, J. Ruiz-Arias, V. Fanego, L. Ramirez, M. Romeo, C. Kurz, L. Pomares, C. Guerrero, Benchmarking of different approaches to forecast solar irradiance, in: 24th European Photovoltaic Solar Energy
Conference, 2009

URL http://www.meteotest.ch/fileadmin/user_upload/ Sonnenenergie/pdf/24th_EU_PVSEC_5BV.2.50_lorenz_final. pdf

[22] A. Zeh, R. Witzmann, Operational strategies for battery storage systems in low-voltage distribution grids to limit the feedin power of roof-mounted solar power systems, Energy Procedia 46 (2014) 114 - 123, 8th International Renewable Energy Storage Conference and Exhibition (IRES 2013). doi:http: //dx.doi.org/10.1016/j.egypro.2014.01.164.

URL http://www.sciencedirect.com/science/article/pii/ S1876610214001805

[23] J. Moshövel, K.-P. Kairies, D. Magnor, M. Leuthold, M. Bost, S. G?hrs, E. Szczechowicz, M. Cramer, D. U. Sauer, Analysis of the maximal possible grid relief from PV-peak-power impacts by using storage systems for increased self-consumption, Applied Energy 137 (2015) 567-575. doi: $10.1016 / j$.apenergy .2014.07.021.

URL http://dx.doi.org/10.1016/j.apenergy.2014.07.021

[24] Y. Riesen, P. Ding, S. Monnier, N. Wyrsch, C. Ballif, Peak shaving capability of household grid-connected pv-system with local storage: A case study, in: 28th European Photovoltaic Solar Energy Conference, 2013.

[25] T. Beck, H. Kondziella, G. Huard, T. Bruckner, Assessing the influence of the temporal resolution of electrical load and PV generation profiles on self-consumption and sizing of PV-battery systems, Applied Energy 173 (2016) 331-342. doi :10.1016/j . apenergy . 2016.04.050. URL http://dx.doi.org/10.1016/j.apenergy.2016.04.050

[26] C. Bucher, J. Betcke, G. Andersson, Effects of variation of temporal resolution on domestic power and solar irradiance measurements, in: ??, 2013.

[27] J. Weniger, T. Tjaden, V. Quaschning, Sizing of residential PV battery systems, Energy Procedia 46 (2014) 78-87. doi : 10.1016/j . egypro. 2014.01 .160 . URL http://dx.doi.org/10.1016/j.egypro.2014.01.160

[28] D. Magnor, N. Soltau, M. Bragard, A. Schmiegel, R. D. Doncker, D. Sauer, Analysis of the model dynamics for the battery and battery converter in a grid connected $5 \mathrm{kw}$ photovoltaic system, in: 25th European Photovoltaic Solar Energy Conference and Exhibition, 2010. URL http://www.sol-ion-project.eu/export/sites/ default/en/_data/publications/mediatheque-files/Conf_ EUPVSEC_Valencia_ISEA_0910.pdf

[29] G. Notton, V. Lazarov, L. Stoyanov, Optimal sizing of a grid-connected pv system for various pv module technologies and inclinations, inverter efficiency characteristics and locations, Renewable Energy 35 (2) (2010) 541 - 554. doi:10.1016/j.renene.2009.07.013.

URL http://www.sciencedirect.com/science/article/pii/ S0960148109003085

[30] Y. Thiaux, L. Schmerber, J. Seigneurbieux, B. Multon, H. B. Ahmed, Comparison between lead-acid and li-ion accumulators in stand-alone photovoltaic system using the gross energy requirement criterion, in: 24th European Photovoltaic Solar Energy Conference, 21-25 September 2009, Hamburg, Germany, 2009.

[31] J. Stein, D. Riley, C. W. Hansen, Pv lib toolbox, Sandia National Laboratories, website: http://pvpmc.org (2015).

[32] N. Wyrsch, Y. Riesen, C. Ballif, Effect of the fluctuations of pv production and electricity demand on the pv electricity self-consumption, in: Proceedings of the 28th European Photovoltaic Solar Energy Conference and Exhibition, p. 4322 - 4324, 2013. 